\title{
Predictive Power of Unconjugated Estriol in Diagnosis of Gestational Diabetes: A Cohort Study
}

\author{
Azam Amirian ${ }^{1}$, Nourossadat Kariman (iD ${ }^{2,}{ }^{*}$, Mehdi Hedayati (iD ${ }^{3}$, Nasrin Borumandnia ${ }^{4}$ and Zohre \\ Sheikhan ${ }^{5}$ \\ ${ }^{1}$ School of Nursing and Midwifery, Shahid Beheshti University of Medical Sciences, Tehran, Iran \\ ${ }^{2}$ Midwifery and Reproductive Health Research Center, Department of Midwifery and Reproductive Health, School of Nursing and Midwifery, Shahid Beheshti University of \\ Medical Sciences, Tehran, Iran \\ ${ }^{3}$ Department of Biochemistry, Cellular and Molecular Research Center, Research Institute for Endocrine Sciences, Shahid Beheshti University of Medical Sciences, Tehran, Iran \\ ${ }^{4}$ Urology Nephrology Research Center, Shahid Beheshti University of Medical Sciences, Tehran, Iran \\ ${ }^{5}$ Midwifery and Reproductive Health Research Center, School of Nursing and Midwifery, Shahid Beheshti University of Medical Sciences, Tehran, Iran \\ Corresponding author: Midwifery and Reproductive Health Research Center, Department of Midwifery and Reproductive Health, School of Nursing and Midwifery, Shahid \\ Beheshti University of Medical Sciences, Tehran, Iran. Email: n_kariman@yahoo.com
}

Received 2019 July 19; Revised 2019 October 21; Accepted 2019 October 23.

\begin{abstract}
Background: Gestational diabetes is the most common antenatal medical complication that is associated with adverse short- and long-term maternal, fetal, and neonatal outcomes. Reducing maternal and fetal complications requires the early diagnosis of gestational diabetes. Unconjugated Estriol (UE) has led to insulin resistance under in vitro conditions.

Objectives: This study aimed to determine the predictive power of unconjugated estriol in the diagnosis of gestational diabetes in Tehran, Iran.

Methods: The present historical cohort study was conducted on 523 pregnant women presenting to two university-affiliated hospitals in Tehran, Iran, 2017-2018. The level of unconjugated estriol was determined at the 14th -17th week of pregnancy, and gestational diabetes was diagnosed at the 24th - 28th week of pregnancy using the oral glucose tolerance test with 75 grams of glucose. Data were collected through interviews and sampling was carried out using a convenience sampling method.

Results: Out of 523 pregnant women examined, 63 (12\%) were placed in the gestational diabetes group and 460 (88\%) in the nongestational diabetes group. The best cutoff point for unconjugated estriol was determined using the ROC curve as $0.965 \mathrm{MOM}$. We obtained $66.66 \%$ sensitivity, $54.78 \%$ specificity, $16.8 \%$ positive predictive value, and 92.30 negative predictive value for the UE test. Conclusions: Given the acceptable sensitivity (66.66\%) and specificity (54.78\%) obtained for the UE test and the area under the ROC curve of 0.60 , it appears that the UE test can be considered a new, accessible, and reliable screening test for gestational diabetes.
\end{abstract}

Keywords: Cohort Studies, Estriol, Fetus, Gestational Diabetes, Glucose, Insulin Resistance, Pregnancy, Unconjugated

\section{Background}

Diabetes is a metabolic disease with a constantly increasing number of affected people such that it will be the seventh cause of death by 2030 (1). Gestational Diabetes Mellitus (GDM) is one of the main forms of diabetes that is defined as varying severities of carbohydrate intolerance that initially begins or is diagnosed during pregnancy (2).

One out of every ten pregnancies worldwide is accompanied by diabetes, and $90 \%$ of these cases are gestational diabetes (3). According to the International Diabetes Federation (IDF) report in 2017, one of every seven births was a product of gestational diabetes (4), and the rate was reported as 5.88\% in Iran (5). With the current increase in the worldwide prevalence of obesity, the prevalence of gesta- tional diabetes is also increasing (6).

Gestational diabetes is associated with adverse shortand long-term maternal, fetal, and neonatal outcomes (2). The most common short-term outcomes include macrosomia, polyhydramnios, preeclampsia, stillbirth, cesarean section, and postpartum hemorrhage $(7,8)$. In the longterm, gestational diabetes is associated with a risk of obesity, glucose intolerance, and neonatal metabolic syndrome (9). In addition, gestational diabetes is a risk factor for the development of type-II diabetes and hypertension in mothers (9).

One of the causes of gestational diabetes is insulin resistance (10). During a normal pregnancy, the increased secretion of diabetogenic placental hormones, including the growth hormone, corticotropin-releasing hormone (CRH), 
placental lactogen, and progesterone, along with maternal hormonal changes lead to insulin resistance and these changes can cause the development of gestational diabetes (11).

The trend of changes leading to gestational diabetes begins during the weeks and months prior to diagnosis, and the factors related to these changes appear in the blood before clinical diagnosis (12). The effectiveness of early-pregnancy interventions has been formerly demonstrated in reducing the risk of developing gestational diabetes and its complications. In fact, pregnancy outcomes can be improved with the early, accurate diagnosis of gestational diabetes $(13,14)$.

After more than 50 years of research, there is still no general consensus on the best method for the screening and diagnosis of gestational diabetes (2). Gestational diabetes is usually diagnosed at the 24th - 28th week of pregnancy. The Oral Glucose Tolerance Test (OGTT) with 75 grams of glucose is currently the standard test for the diagnosis of gestational diabetes (15). Nevertheless, this test is associated with some problems, such as the patient's need for fasting prior to the test, heavy costs, time-consuming nature of the test, conflicting results in people from different races and ethnicities, and some patients' intolerance to high amounts of powdered sugar. Some studies have proposed using 50 grams of glucose in the OGTT for the diagnosis of gestational diabetes (16), but this test is limited in terms of sensitivity and specificity (17). Moreover, the OGTT is unable to diagnose mild glucose intolerance, and this deficiency could cause perinatal complications (18). Studies investigating the predictive power of fasting plasma glucose for gestational diabetes have concluded that fasting plasma glucose in early pregnancy is an ineffective screening measure for gestational diabetes (19). Moreover, despite the serious complications of gestational diabetes, its diagnostic test is carried out during late pregnancy, when it is too late to take preventive measures for the condition $(20,21)$. In addition, recent evidence suggests that gestational diabetes can be prevented by interventions performed in early pregnancy. Therefore, a simple and effective strategy in early pregnancy is required (22).

Many clinical researchers are interested in studying changes in the biochemical markers that are routinely screened during pregnancy to detect adverse pregnancy outcomes early on and mitigate their complications (20). Unconjugated Estriol(UE3) is one of the biochemical markers assessed in the screening of Down's syndrome in the early second trimester of pregnancy (2) and has been considered by researchers as a new diagnostic technique for gestational diabetes (20). Estrogen is a steroid hormone that regulates glucose homeostasis by increasing insulin sensitivity, glucose-stimulated insulin secretion, and glucose transfer expression (22). In addition, postmenopausal estrogen replacement has reduced the risk of developing type II diabetes (23). Estrogen and its receptor are known as insulin sensitivity regulators (24). Meanwhile, estriol is a weak estrogen agonist that has a strong antagonistic activity along with estradiol (25). Unconjugated Estriol (UE) has led to insulin resistance under in vitro conditions (26). Moreover, the low levels of maternal serum UE in the second trimester have been associated with adverse pregnancy complications (27).

Few studies have investigated the predictive power of UE in gestational diabetes. A study conducted in 2017 showed a significant relationship between increased levels of UE and gestational diabetes (28). In contrast, another study reported reduced UE levels in women with gestational diabetes (29). The results of another study showed no relationships between UE levels and gestational diabetes (27).

The diagnosis of gestational diabetes using a test with high sensitivity and specificity and the prevention of its complications play major roles in improving maternal and neonatal health. Moreover, the evaluation of UE in the early second trimester can be used as a new and accessible marker of gestational diabetes.

\section{Objectives}

The present study was conducted to determine the predictive power of UE in diagnosing gestational diabetes in Tehran, Iran.

\section{Methods}

\subsection{Study Design and Sampling Criteria}

The present historical cohort study was conducted on 523 pregnant women who referred to the prenatal care clinics of Mahdieh and Taleghani hospitals in Tehran, capital of Iran, in 2017- 2018.

Data were collected using a two-part form. The first part was to assess the inclusion criteria and the second part to gather demographic details, midwifery history, the checklist recording, the UE levels in weeks 14 - 17 of pregnancy, and the results of the gestational diabetes screening test with 75 grams of oral glucose in weeks $24-28$ of pregnancy. Gestational diabetes was assessed based on the WHO instructions. The two-hour oral glucose tolerance test was performed in all the participants after taking 75 grams of glucose in weeks $24-28$ of pregnancy ( 2 and 15). Fasting blood glucose of 92 or higher, blood glucose of 180 or higher after one hour of taking glucose, and 153 
or higher after two hours of taking glucose were considered abnormal. The diagnosis of gestational diabetes was definitive, even with one abnormal blood glucose result (2, 15).

Sampling was of the multistage type. In the first stage, centers with the highest statistics of prenatal care referrals were selected from among all teaching hospitals affiliated to the Shahid Beheshti University of Medical Sciences. Then, a quota was given to each center based on the total number of pregnant women presenting to the center. Finally, purposive sampling was conducted in each center and continued until the required sample size was achieved.

\subsection{Inclusion and Exclusion Criteria}

The inclusion criteria included women with singleton pregnancy aged 20 - 40 years with a gestational age of 24 34 weeks (based on the first day of the last menstruation or the first-trimester ultrasound). The exclusion criteria were a history of known systemic diseases (i.e., diabetes, preeclampsia, hypertension, PCO, etc.), type II diabetes in first-degree relatives, BMI $>30 \mathrm{~kg} / \mathrm{m}^{2}$, a history of stillbirth, habitual abortion, fetal anomalies, fetal macrosomia, intake of medications affecting glucose metabolism, smoking, and drug use.

\subsection{Sample Size}

The sample size was determined as 523 women based on the prevalence of gestational diabetes of $6 \%$, the type I error of $\alpha=0.05$, required sensitivity of $80 \%\left(S^{\mathrm{e}}\right)$, and the required accuracy of $d=0.1$.

\subsection{Data Collection Tools}

The data collection tools included a demographic and obstetrics questionnaire, a blood glucose kit/device, and a UE kit/device. The validity of the demographic and midwifery questionnaire was assessed using the content validity method. In both centers, the blood sugar level was determined using Pars Azmun kits, Hitachi Auto-Analyzer 911 (made in Japan), and glucose oxidase method. The validity of the UE kit was measured based on the kit's manufacturer (i.e., LDN Co., Germany) in both centers. This test was conducted using the ELISA method by utilizing an ELISA reader (Hyperion Co., France). To ensure the reliability of the UE and blood glucose measuring devices, they were calibrated every morning by medical engineers. All UE and blood sugar tests were carried out by the same devices and the same laboratory technicians in both centers. Concurrent observation (inter-rater consistency) was used to determine the reliability of the technicians. For this purpose, ten blood sugar samples and 10 UE samples were concurrently tested by the two similarly-experienced technicians. The results were assessed using Pearson's correlation test (Pearson correlation coefficient $=0.95$ ).

\subsection{Data Collection}

The participants' data were collected by an expert midwife. After explaining the study objectives and obtaining the informed consent of pregnant women who had visited these centers at their 24th - 34th week of pregnancy and met the inclusion criteria (ensured through the completion of the first part of the data form), the second part of the data form (demographic and midwifery details) was completed. The results of the UE level test at the 14th - 17th week of pregnancy conducted at the two centers' laboratories were recorded in the relevant checklist. We also used the relevant checklist to record the results of the screening test for gestational diabetes, which was carried out using the OGTT with 75 grams of glucose at the 24th - 28th week of pregnancy as per the center's routine. After reaching the required sample size, i.e., 523 pregnant women, the relationship between different values of UE and gestational diabetes was assessed by using statistical methods and the best UE cutoff point was determined. Then, the participants were divided into two groups based on the cutoff point. The statistical relationship between UE and the incidence of gestational diabetes was then assessed in the two groups.

\subsection{Data Analysis}

Data were analyzed using IBM SPSS Statistics Software, version 20 (IBM Corp., Armonk, N.Y., USA) and R software. Frequency tables were drawn using descriptive statistics at a confidence interval of $95 \%$. The differences between the groups with and without gestational diabetes were assessed using the independent $t$-test for quantitative variables, the chi-square test for qualitative variables, the Mann-Whitney test for ordinal variables, and logistic regression. The ROC curve was used to determine the UE threshold and to determine the sensitivity and specificity of the UE test. Meanwhile, the UE optimal cutoff point for the prediction of gestational diabetes was obtained using the Youden index (J).

\section{Results}

Out of 523 participants, 63 were assigned to the gestational diabetes group and 460 to the non-gestational diabetes group. There were no significant differences between the participants in terms of education, occupation, housing status, gestational age, gravidity, parity, and the number of abortions. 
Table 1 presents the confounding variables and the demographic and midwifery details. The mean \pm standard deviation of serum UE was $1.15 \pm 0.60$ multiples of the median (MOM) in the gestational diabetes group and $1.05 \pm$ $0.58 \mathrm{MOM}$ in the control group. The lowest and the highest serum UE levels were $0.49 \mathrm{MOM}$ and $4.05 \mathrm{MOM}$ in the gestational diabetes group and 0.01 MOM and 5.06 MOM in the control group, respectively; therefore, a significant difference was observed between the two groups in terms of serum UE level $(\mathrm{P}=0.01)$.

The diagnosis of gestational diabetes was made by assessing the UE level using the ROC curve (Figure 1).

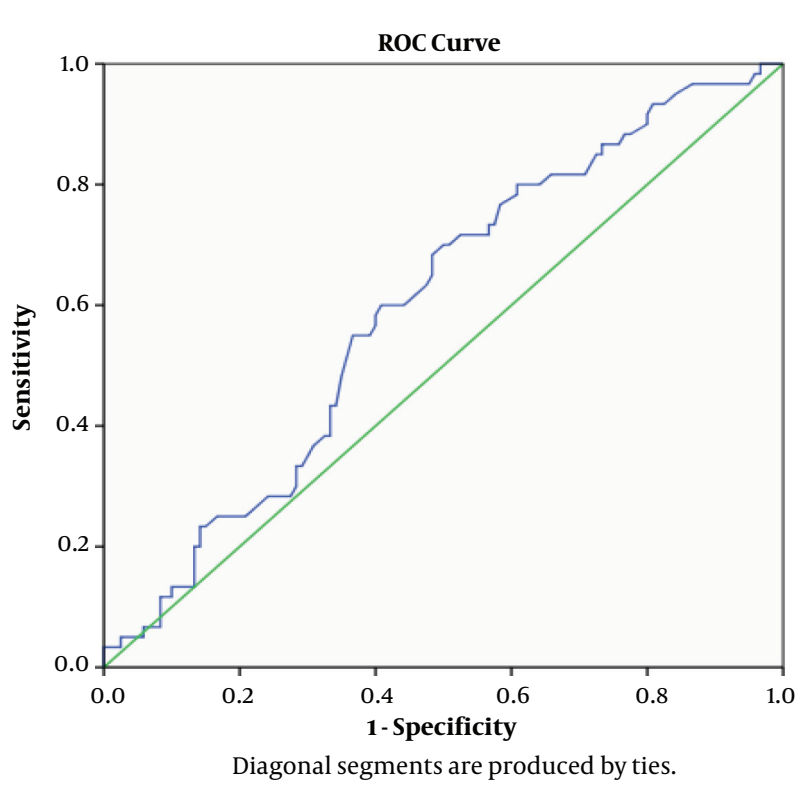

Figure 1. The UE curve for the prediction of gestational diabetes in the study participants

Using the ROC curve and after determining the minimum and maximum threshold levels of UE (Table 2), the appropriate UE threshold level for the diagnosis of gestational diabetes was obtained as $0.965 \mathrm{MOM}$. The area under the ROC curve was 0.60 , which indicated the acceptability of the UE test for predicting gestational diabetes.

Based on the results of the ROC curve and the threshold value of $0.965 \mathrm{MOM}$, the UE test turned out positive for 42 participants (66.7\%) in the gestational diabetes group and 208 (45.22\%) in the non-gestational diabetes group (Table 3). According to the data presented in Table 3, this test had $66.66 \%$ sensitivity, $54.78 \%$ specificity, $16.8 \%$ positive predictive value, $92.30 \%$ negative predictive value, 1.21 positive likelihood ratio, and 0.73 negative likelihood ratio. The cutoff point was 0.97 estimated by the Youden index. The sensitivity, specificity, positive and negative pre- dictive values, DLR-positive, DLR-negative, and optimal criterion were $67 \%, 55 \%, 17 \%, 92 \%, 1.47,0.61$, and 0.21 in detecting gestational diabetes by evaluating UE of the early second trimester of pregnancy, respectively.

The confounding variables (BMI and mother's age) were controlled using the logistic regression analysis, which showed that the odds of developing gestational diabetes increased by $18 \%$ per each unit of increase in BMI and by $5 \%$ per each year of increase in age. After controlling for confounding variables, the odds of developing gestational diabetes increased 2.67 times with each unit increase in the UE level (Table 4).

\section{Discussion}

The present findings showed that the UE test had an acceptable predictive power for diagnosing gestational diabetes. Maternal UE levels can be used to predict fetal adaptation and health (25). Unconjugated Estriol (UE) is currently used as a component of Down syndrome diagnostic tests in pregnancy (30). Moreover, the serum UE concentration is associated with factors such as the mother's weight, gestational age, and race (31).

Limited studies have been conducted on the predictive power of estriol as an indicator of gestational diabetes. In line with the present findings, Hur et al. (2017) investigated the relationship between serum UE levels and gestational diabetes in South Korea and showed that the UE levels of $>2 \mathrm{MOM}$ in the early second trimester had a significant relationship with the development of gestational diabetes (20).

In a study conducted by Settiyanan et al. (2016) in Thailand to determine the relationship between abnormal UE values at the 15th -21st week of pregnancy and adverse pregnancy outcomes, no significant relationship was found between UE levels and gestational diabetes, which is inconsistent with the present findings. This difference can be explained in a number of ways. First, in Settiyanan et al. study, participants were grouped using UE percentiles (normal, percentile $\leq 5$, and percentile $\geq 95$ ), but in the present study, grouping was based on the cutoff point of the study population and using the ROC curve. Besides, Settiyanan et al. study did not discuss their method of diagnosing gestational diabetes while differences in diagnostic methods can directly affect the study results. Other possible reasons for the disparity between the study findings could be the racial differences, as race dictates different rates of gestational diabetes in different countries (28).

Unlike the present study, in a study conducted in Turkey, Sayin et al. (2008) found a relationship between lower UE levels and gestational diabetes. Their study was conducted to investigate the results of a triple marker 


\begin{tabular}{|c|c|c|c|c|}
\hline Group/Characteristic & Women with Gestational Diabetes $(\mathrm{N}=63)$ & Control Group $(N=460)$ & Statistical Test & P Value \\
\hline Age, $y$ & $30.2 \pm 4.78$ & $5.38 \pm 28.56$ & Independent $t$ & 0.022 \\
\hline Education (high school) & $37(58.7)$ & $268(58.3)$ & Mann-Whitney & 0.128 \\
\hline Mother's job (including housewifery) & $57(90.5)$ & $426(92.6)$ & Chi-square & 0.550 \\
\hline Spouse's job (self-employed) & $44(69.8)$ & $369(80.2)$ & Chi-square & 0.146 \\
\hline Gestational age & $31.25 \pm 4.36$ & $3.47 \pm 32.41$ & Independent $t$ & 0.142 \\
\hline Gravidity & $2.00(1.00-3.00)$ & $2.00(1.00-3.00)$ & Mann-Whitney & 0.486 \\
\hline Parity & $1.00(1.00-2.00)$ & $1.00(1.00-2.00)$ & Mann-Whitney & 0.591 \\
\hline Number of abortions & $1.00(1.00)$ & $1.00(1.00)$ & Mann-Whitney & 0.273 \\
\hline First-trimester BMI & $25.98 \pm 2.57$ & $3.26 \pm 24.38$ & Independent $t$ & 0.000 \\
\hline Housing status (renting) & $43(68.3)$ & $325(70.7)$ & Chi-square & 0.703 \\
\hline
\end{tabular}

${ }^{\mathrm{a}}$ Values are expressed as mean $\pm \mathrm{SD}$, No. (\%), or median (IQR).

\begin{tabular}{lcc}
\hline $\begin{array}{l}\text { Table 2. Sensitivity and Specificity of Serum UE Levels Based on Different Cutoff Val- } \\
\text { ues in Study Participants }\end{array}$ & Sensitivity & Specificity \\
\hline Unconjugated Estriol & 0.683 & 0.496 \\
\hline $\mathbf{0 . 9 2 5}$ & 0.683 & 0.483 \\
\hline $\mathbf{0 . 9 3 5}$ & 0.683 & 0.476 \\
$\mathbf{0 . 9 4 5}$ & 0.667 & 0.463 \\
\hline $\mathbf{0 . 9 5 5}$ & 0.667 & 0.452 \\
\hline $\mathbf{0 . 9 6 5}$ & 0.635 & 0.443 \\
\hline $\mathbf{0 . 9 7 5}$ & 0.619 & 0.426 \\
\hline $\mathbf{0 . 9 8 5}$ & 0.603 & 0.411 \\
\hline $\mathbf{0 . 9 9 5}$ & 0.587 & 0.393 \\
\hline $\mathbf{1 . 0 0 5}$ & & \\
\hline
\end{tabular}

Table 3. Relative Frequency of Serum UE Based on the Threshold Value (the Cutoff Point) of 0.965 MOM in the Gestational Diabetes and Control Groups

\begin{tabular}{lcc}
\hline Unconjugated Estriol & $\begin{array}{c}\text { Women with } \\
\text { Gestational Diabetes }\end{array}$ & $\begin{array}{c}\text { Women without } \\
\text { Gestational Diabetes } \\
\text { (Control Group) }\end{array}$ \\
\hline $\mathbf{0 . 9 6 5} \geq$ & $21(33.3)$ & $252(54.78)$ \\
\hline $\mathbf{0 . 9 6 5}<$ & $42(66.7)$ & $208(45.22)$ \\
\hline Total & $63(100)$ & $460(100)$ \\
\hline
\end{tabular}

Table 4. Determining the Odds of Developing Gestational Diabetes in Terms of Some Risk Factors Using the Logistic Regression

\begin{tabular}{lccc}
\hline Variable & P Value & Odds Ratio & Confidence Interval \\
\hline UE & 0.001 & 2.67 & $1.51-4.73$ \\
BMI & 0.001 & 1.18 & $1.07-1.30$ \\
Age & 0.049 & 1.05 & $1-1.11$ \\
\hline
\end{tabular}

screening test at the 16 th - 18 th week of pregnancy, and using the ROC curve, the threshold for the diagnosis of gesta- tional diabetes was measured as $0.88 \mathrm{MOM}$ and the area under the ROC curve was measured as $57 \%$. They reported UE test sensitivity of $36.2 \%$ and specificity of $78.5 \%$. The possible reasons for the differences in results can be the diagnosis of gestational diabetes using the OGTT with 100 grams of glucose and determining the UE cutoff point using MedCalc V.4.30. In the present study, the cutoff point was found using SPSS V. 20 and R software. Furthermore, Sayin et al. study was retrospective and aimed to investigate several adverse pregnancy outcomes, which may have reduced the accuracy of the study, as not all the confounding factors of gestational diabetes were controlled. Moreover, in Sayin et al. study, the UE level was measured by radioimmunoassay, but the present study used the ELISA method (29).

During pregnancy, the maternal hormones are regulated by the pituitary gland and the placenta and these hormones have major roles in the mother's development of insulin resistance (32). Estrogen receptors are expressed in the pancreatic Langerhans islet beta cells and beta-17 estradiol is also associated with the increased biosynthesis and secretion of insulin (33). Moreover, estriol has an antiestrogenic activity together with estradiol (34). Estriol inhibits the bond between estradiol and estrogen receptors (35). In an in vitro environment, estriol is known as an insulin resistance factor(26). Consequently, increased estriol in pregnancy could likely be associated with insulin resistance.

The UE test is often performed in the early second trimester to screen for Down syndrome. It removes the need for incurring costs and running tests in research centers for the diagnosis of gestational diabetes. Meanwhile, the OGTT is carried out at the 24th - 28th week of pregnancy at extra costs and has a poor predictive power in early pregnancy (19). Given the increasing prevalence of gestational 
diabetes in Iran and across the world and the need for its early diagnosis to reduce adverse maternal and fetal complications, a convenient marker such as UE examined in early pregnancy can be extremely helpful.

The strengths of this study include the use of the latest gestational diabetes diagnostic criteria, controlling for confounding variables, the use of R software in conjunction with SPSS, and the use of the Youden index for determining the best cutoff point. In addition, all the participants underwent the two-hour glucose tolerance test for the diagnosis of gestational diabetes by taking 75 grams of oral glucose. The study limitations include recording the patients' disease history in a self-report way, which could be associated with misunderstandings about the medical status along with the UE level.

\subsection{Conclusions}

Given the need for the prevention and early diagnosis of gestational diabetes to reduce the resultant maternal and fetal complications, UE can be used as a gestational diabetes predictive factor. Measures should be taken to train all health service providers in this regard so that the risk of gestational diabetes is taken into account when noticing an increase in UE levels in the test results of pregnant women; therefore, immediate preventive and medical measures can be taken to reduce the complications. Since the prevalence of gestational diabetes is rising, further prospective studies with larger sample sizes are recommended for achieving more powerful results.

\section{Acknowledgments}

The present study was derived from a master of midwifery thesis. We, hereby, wish to express our gratitude to all participating pregnant women, to the research deputy of Shahid Beheshti University of Medical Sciences for granting and support of the study, and to the faculty and director of the Midwifery Department of the School of Nursing and Midwifery of Shahid Beheshti University of Medical Sciences for recognizing the need for conducting this research.

\section{Footnotes}

Authors' Contribution: Study concept and design and analysis and interpretation of data: Azam Amirian, Nourossadat Kariman, Nasrin Borumandnia, Mehdi Hedayati, and Zohre Sheikhan; drafting of the manuscript: Azam Amirian and Nourossadat Kariman; critical revision of the manuscript for important intellectual content:
Azam Amirian, Nourossadat Kariman, and Nasrin Borumandnia; statistical analysis: Azam Amirian and Nasrin Borumandnia.

Conflict of Interests: There are no conflicts of interest to disclose.

Ethical Approval: This study was approved by the Research Ethics Committee of Shahid Beheshti University of Medical Sciences, Tehran, Iran (code number: IR.SBMU.PHNM.1396.777).

Funding/Support: The Research Deputy of Shahid Beheshti University of Medical Sciences supported the study.

Patient Consent: After explaining the study objectives, informed consent was obtained from all participants.

\section{References}

1. World Heath Organization. Fact sheet. WHO 2017. 2017. Available from: http://www.who.int/mediacentre/factsheets/fs312/en/.

2. Cunningham F, Leveno K, Bloom S, Spong CY, Dashe J. Williams obstetrics. 25 ed. New York: Mcgraw-Hill; 2018.

3. World Heath Organization. Pocket book of hospital care for mothers. 2017. Available from: https://apps.who.int/iris/handle/10665/ 258716.

4. Cho NH, Shaw JE, Karuranga S, Huang Y, da Rocha Fernandes JD, Ohlrogge AW, et al. IDF Diabetes Atlas: Global estimates of diabetes prevalence for 2017 and projections for 2045. Diabetes Res Clin Pract. 2018;138:271-81. doi:10.1016/j.diabres.2018.02.023. [PubMed: 29496507].

5. Almasi SZ, Salehiniya $\mathrm{H}$. [The prevalence of gestational diabetes mellitus in Iran (1993-2013): A systematic review]. J Isfahan Med Sch. 2014;32(299):1396-412. Persian.

6. Aaron B. Gestational diabetes mellitus: Obstetrical issues and management. Uptodate; 2017. Available from: https://www.uptodate.com/ contents/search?search=Gestational $\% 20$ diabetes $\% 20$ mellitus: $\% 20$ Obstetrical $\% 20$ issues $\% 20$ and $\% 20$ management $\& x=9 \& y=10$.

7. Billionnet C, Mitanchez D, Weill A, Nizard J, Alla F, Hartemann A, et al. Gestational diabetes and adverse perinatal outcomes from 716,152 births in France in 2012. Diabetologia. 2017;60(4):636-44. doi: 10.1007/s00125-017-4206-6. [PubMed: 28197657]. [PubMed Central: PMC6518373].

8. Sweeting AN, Ross GP, Hyett J, Molyneaux L, Constantino M, Harding AJ, et al. Gestational diabetes mellitus in early pregnancy: Evidence for poor pregnancy outcomes despite treatment. Diabetes Care. 2016;39(1):75-81. doi: 10.2337/dc15-0433. [PubMed: 26645084].

9. Damm P, Houshmand-Oeregaard A, Kelstrup L, Lauenborg J, Mathiesen ER, Clausen TD. Gestational diabetes mellitus and long-term consequences for mother and offspring: A view from Denmark. Diabetologia. 2016;59(7):1396-9. doi: 10.1007/s00125-016-3985-5. [PubMed: 27174368].

10. American Diabetes Association. Management of diabetes in pregnancy. Diabetes Care. 2016;39 Suppl 1:S94-8. doi: 10.2337/dc16-S015. [PubMed: 26696688].

11. Petraglia F, D'Antona D, Snyder PJ. Maternal adaptations to pregnancy: Endocrine and metabolic changes. UpToDate; 2017. Available from: https://www.uptodate.com/contents/maternal-adaptations-topregnancy-endocrine-and-metabolic-changes.

12. Beneventi F, Simonetta M, Lovati E, Albonico G, Tinelli C, Locatelli E, et al. First trimester pregnancy-associated plasma protein-A in pregnancies complicated by subsequent gestational diabetes. Prenat $\mathrm{Di}$ agn. 2011;31(6):523-8. doi: 10.1002/pd.2733. [PubMed: 21404306]. 
13. Petrella E, Tamborrino V, Di Cerbo L, Neri I, Facchinetti F. An early, customized low-glycemic-index diet prevents adverse pregnancy outcomes in overweight/obese women. Minerva Ginecol. 2018;70(3):25460. doi: 10.23736/S0026-4784.17.04156-9. [PubMed: 29083138].

14. Zandinava H, Sehhatti Shafaei F, Mohammad-Alizadeh Charandabi S, Ghanbari Homayi S, Mirghafourvand M. Effect of educational package on self-care behavior, quality of life, and blood glucose levels in pregnant women with gestational diabetes: A randomized controlled trial. Iran Red Crescent Med J. 2017;19(4). doi: 10.5812/ircmj.44317.

15. World Heath Organization. Diagnostic criteria and classification of hyperglycaemia first detected in pregnancy. WHO; 2013. Available from: https://www.who.int/diabetes/publications/Hyperglycaemia_In_ Pregnancy/en/.

16. Kleinwechter H, Schafer-Graf U, Buhrer C, Hoesli I, Kainer F, KautzkyWiller A, et al. Gestational diabetes mellitus (GDM) diagnosis, therapy and follow-up care: Practice Guideline of the German Diabetes Association(DDG) and the German Association for Gynaecologyand Obstetrics (DGGG). Exp Clin Endocrinol Diabetes. 2014;122(7):395-405. doi: 10.1055/s-0034-1366412. [PubMed: 25014091].

17. Lekva T, Godang K, Michelsen AE, Qvigstad E, Normann KR, Norwitz ER, et al. Prediction of gestational diabetes mellitus and pre-diabetes 5 years postpartum using $75 \mathrm{~g}$ oral glucose tolerance test at 14-16 weeks' gestation. Sci Rep. 2018;8(1):13392. doi:10.1038/s41598-018-31614z. [PubMed: 30190548]. [PubMed Central: PMC6127333].

18. Sacks DA, Chen W, Wolde-Tsadik G, Buchanan TA. Fasting plasma glucose test at the first prenatal visit as a screen for gestational diabetes. Obstet Gynecol. 2003;101(6):1197-203. doi: 10.1016/s00297844(03)00049-8. [PubMed: 12798525].

19. Metzger BE, Gabbe SG, Persson B, Buchanan TA; International Association of Diabetes; Pregnancy Study Groups Consensus Panel, et al. International association of diabetes and pregnancy study groups recommendations on the diagnosis and classification of hyperglycemia in pregnancy. Diabetes Care. 2010;33(3):676-82. doi:10.2337/dc09-1848. [PubMed: 20190296]. [PubMed Central: PMC2827530].

20. Hur J, Cho EH, Baek KH, Lee KJ. Prediction of gestational diabetes mellitus by unconjugated estriol levels in maternal serum. Int J Med Sci. 2017;14(2):123-7. doi:10.7150/ijms.17321. [PubMed: 28260987]. [PubMed Central: PMC5332840].

21. Falcone V, Kotzaeridi G, Breil MH, Rosicky I, Stopp T, YerlikayaSchatten G, et al. Early assessment of the risk for gestational diabetes mellitus: Can fasting parameters of glucose metabolism contribute to risk prediction? Diabetes Metab J. 2019;43(19). doi: 10.4093/dmj.2018.0218. [PubMed:30877716].

22. Sweeting AN, Wong J, Appelblom H, Ross GP, Kouru H, Williams $\mathrm{PF}$, et al. A novel early pregnancy risk prediction model for gestational diabetes mellitus. Fetal Diagn Ther. 2019;45(2):76-84. doi: 10.1159/000486853. [PubMed: 29898442].

23. Riant E, Waget A, Cogo H, Arnal JF, Burcelin R, Gourdy P. Estrogens protect against high-fat diet-induced insulin resistance and glucose intolerance in mice. Endocrinology. 2009;150(5):2109-17. doi: 10.1210/en.2008-0971. [PubMed: 19164473].

24. Bitoska I, Krstevska B, Milenkovic T, Subeska-Stratrova S, Petrovski
G, Mishevska SJ, et al. Effects of hormone replacement therapy on insulin resistance in postmenopausal diabetic women. Open Access Maced J Med Sci. 2016;4(1):83-8. doi: 10.3889/oamjms.2016.024. [PubMed: 27275336]. [PubMed Central: PMC4884259].

25. Meyer MR, Clegg DJ, Prossnitz ER, Barton M. Obesity, insulin resistance and diabetes: Sex differences and role of oestrogen receptors. Acta Physiol (Oxf). 2011;203(1):259-69. doi: 10.1111/j.1748-1716.2010.02237.x. [PubMed: 21281456]. [PubMed Central: PMC3110567].

26. Falah N, Torday J, Quinney SK, Haas DM. Estriol review: Clinical applications and potential biomedical importance. Clin ResTrial. 2015;1(2):29-33. doi:10.15761/crt.1000109.

27. Collison M, Campbell IW, Salt IP, Dominiczak AF, Connell JM, Lyall $\mathrm{H}$, et al. Sex hormones induce insulin resistance in 3T3-L1 adipocytes by reducing cellular content of IRS proteins. Diabetologia. 2000;43(11):1374-80. doi: 10.1007/s001250051541. [PubMed: 11126405].

28. Settiyanan T, Wanapirak C, Sirichotiyakul S, Tongprasert F, Srisupundit K, Luewan S, et al. Association between isolated abnormal levels of maternal serum unconjugated estriol in the second trimester and adverse pregnancy outcomes. J Matern Fetal Neonatal Med. 2016;29(13):2093-7. doi: 10.3109/14767058.2015.1075503. [PubMed: 27480207].

29. Sayin NC, Canda MT, Ahmet N, Arda S, Sut N, Varol FG. The association of triple-marker test results with adverse pregnancy outcomes in low-risk pregnancies with healthy newborns. Arch Gynecol Obstet. 2008;277(1):47-53. doi: 10.1007/s00404-007-0421-6. [PubMed: 17653738].

30. Cuckle H, Pergament E, Benn P. Maternal serum screening for chromosomal abnormalities and neural tube defects. Genetic disorders and the fetus: diagnosis, prevention and treatment. Chichester, United Kingdom: Wiley; 2016. p. 483-540. doi: 10.1002/9781118981559.ch12.

31. Wanapirak C, Sirichotiyakul S, Luewan S, Yanase Y, Traisrisilp K, Tongsong T. Different median levels of serum triple markers in the second trimester of pregnancy in a Thai Ethnic Group.J Obstet Gynaecol Res. 2012;38(4):686-91. doi: 10.1111/j.1447-0756.2011.01769.x. [PubMed: 22380630].

32. Aguayo-Mazzucato C, van Haaren M, Mruk M, Lee TJ, Crawford C, Hollister-Lock J, et al. Beta cell aging markers have heterogeneous distribution and are induced by insulin resistance. Cell Metab. 2017;25(4):898-910 e5. doi: 10.1016/j.cmet.2017.03.015. [PubMed: 28380379]. [PubMed Central: PMC5471618].

33. Nadal A, Alonso-Magdalena P, Soriano S, Ropero AB, Quesada I. The role of oestrogens in the adaptation of islets to insulin resistance. J Physiol. 2009;587(Pt 21):5031-7. doi: 10.1113/jphysiol.2009.177188. [PubMed: 19687125]. [PubMed Central: PMC2790246].

34. Melamed M, Castano E, Notides AC, Sasson S. Molecular and kinetic basis for the mixed agonist/antagonist activity of estriol. Mol Endocrinol. 1997;11(12):1868-78. doi: 10.1210/mend.11.12.0025. [PubMed: 9369454].

35. Sasson S, Notides AC. Estriol and estrone interaction with the estrogen receptor. II. Estriol and estrone-induced inhibition of the cooperative binding of $[3 \mathrm{H}]$ estradiol to the estrogen receptor. J Biol Chem. 1983;258(13):8118-22. [PubMed: 6863280]. 